\title{
INFRARED SPECTROSCOPY \\ OF BE/X-RAY BINARIES
}

\author{
C. EVERALL, M.J.COE and P. ROCHE \\ High Energy Astrophysics Group, Physics Dept., University of Southampton, \\ Southampton, SO9 5NH, U.K. \\ and
}

A. J. NORTON and S. J. UNGER

The Open University, Physics Dept., Walton Hall, Milton Keynes, MK7 6AA, U. K.

\section{Introduction}

We present infrared spectra of $4 \mathrm{Be} / \mathrm{X}$-ray binaries in the $\mathrm{K}$ band, and 4 spectra in the J, H and $\mathrm{K}$ bands of 2 more sources. The HI IR emission lines are useful determinators of the conditions in the inner regions of the circumstellar disk about the Be star, due to optical depth effects. These are preliminary results, and hope to be followed up by high resolution echelle spectra, where we wish to estimate the velocity field, temperature and density structure of the circumstellar material.

\section{Initial $\mathbf{K}$ band spectra}

The spectra shown in Fig 1, of $4 \mathrm{Be} / \mathrm{X}$-ray binaries (H0521+37, A0535+26, $\mathrm{X}$ Per and 4U0728-25) were take on January 13th 1992 in service time using the $75 / \mathrm{mm}^{-1}$ grating at short focal length with CGS4 on UKIRT. The resolution was $\lambda / \Delta \lambda \sim 350$. Problems with tracking produced ripples that were difficult to correct. The spectra were combined to reduce this effect and are shown with line identifications in Fig. 2. The Pfund series was close to the case B ratios predicted by Hummer and Storey (1987), whereas Br $\gamma$ must have a large optical depth. $\mathrm{NaI}$ emission could only arise in conditions cooler than the temperatures necessary for the free-free emission in the disk. Further details are available in Everall et al. (1993)

\section{J,H,K spectra}

The spectra shown in Figs 3- 6 were take on August 4-5th 1993 using the $75 / \mathrm{mm}^{-1}$ grating at long focal length with CGS4 on UKIRT. The resolution was $\lambda / \Delta \lambda \sim 700$ at $\mathrm{K}$. The targets were $2 \mathrm{Be} / \mathrm{X}$-ray binaries, EXO2030+375 and $4 \mathrm{U} 2204+56$, and $4 \mathrm{U} 1907+09$, an OB supergiant $\mathrm{X}$-ray binary. Absorption lines in the $\mathrm{H}$ band standard means the $\mathrm{H}$ band data is dubious. 
Figure 1:
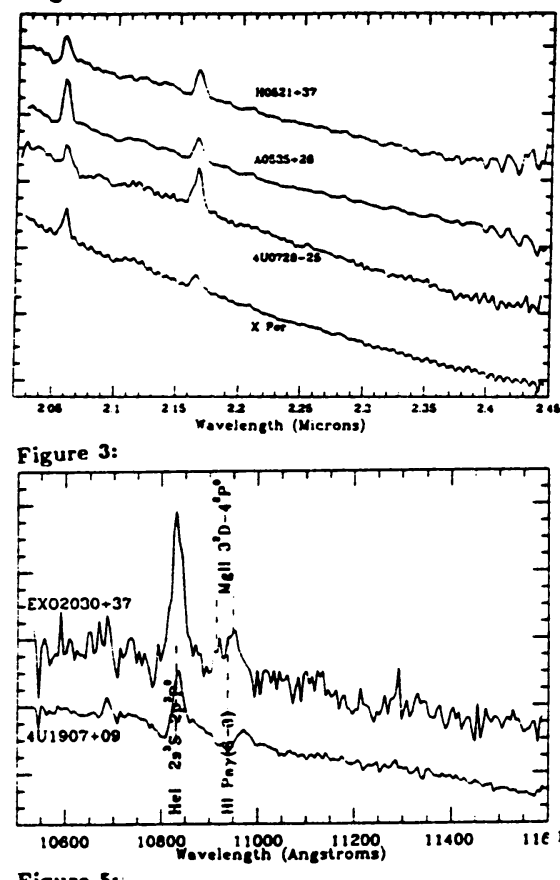

Figure 5:

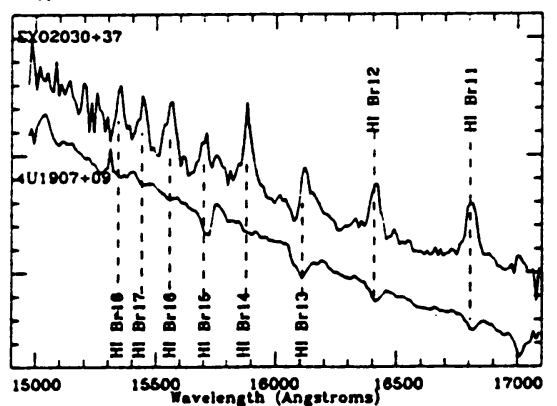

Figure 2:

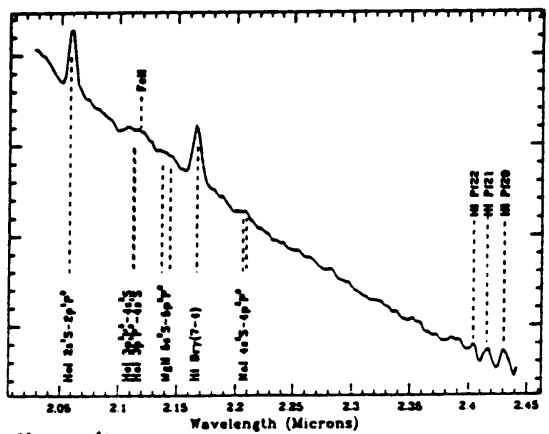

Figure 4:

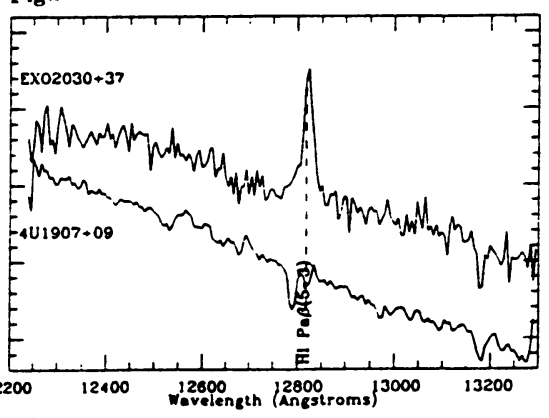

Figure 6:

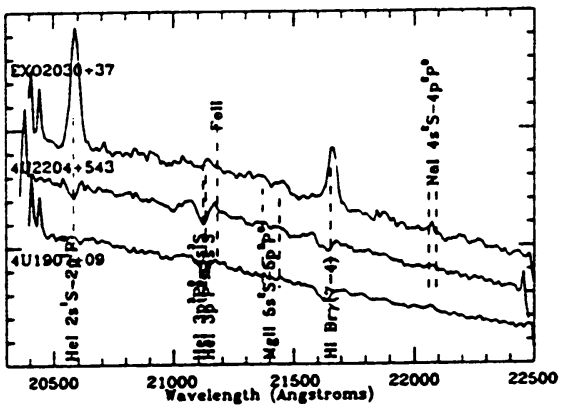

The spectra of $4 \mathrm{U} 1907+09$ varied greatly from the Be star's, with only the HeI $1.08 \mu \mathrm{m}\left(2 \mathrm{~s}^{3} \mathrm{~S}-2 \mathrm{p}^{3} \mathrm{P}^{0}\right)$ line in emission. $4 \mathrm{U} 2204+56$ had no emission lines, and therefore is likely to be in a diskless state. The spectra of EXO2030+375 had many HI emission lines, with a possible turnover in the Brackett series from optically thick to thin emission above $\mathrm{Br} 14$. The $\mathrm{Pa} \beta, \mathrm{Pa} \gamma$ and $\mathrm{Br} \gamma$ lines were all optically thick. These results will be discussed fully in a future publication (Everall et al. 1994).

\section{References}

Everall C. et al. 1993, MNRAS 262, 57-62

Everall C. et al. 1994 (in prep.)

Hummer D., Storey, P. 1987, MNRAS, 224, 801.

McGregor P. J., Hyland A. R., Hillier D. 1988, ApJ, 324, 1071 